\title{
Infrared Skin-Like Active Stretchable Electronics Based on Organic-Inorganic Composite Structures for Promotion of Cutaneous Wound Healing
}

\author{
Lijuan Zhang, Xiaoxiao Jiang, Wei Jiang, Shuang Li, Yuxi Chi, Hao Liu, Maoyi Zhang, \\ Juyao Li, Min Fang, Bing Pan, Yuli Chen, Chuanan Shen, Xu Guo, Rui Li,* Liang Guo,* \\ and Yewang Su*
}

Recent studies on flexible/stretchable medical electronics are almost all focusing on passive monitoring of physiological signs for medical diagnostics, but rare reports have been found on those for active therapeutic purposes. Here, a novel infrared skin-like active stretchable electronics (ISASE) is introduced with the organic-inorganic composite design for promotion of cutaneous wound healing, which can be conformally mounted anywhere on the human body due to its excellent flexibility and stretchability. Comprehensive experiments, including the proliferation and migration of human skin fibroblasts and rat dermal fibroblasts in vitro, and various tests on wound healing of rats, show favorable effects of the ISASE on promoting the fibroblast migration and proliferation, reducing the inflammatory phase, stimulating the angiogenesis, and shorting the wound healing period. A typical ISASE significantly shortens the cure time for a wounded rat by at least two days compared with the normal treatment with 12 days cure. The mechanical tests confirm the validity of the ISASE when used in extreme large deformations (e.g., stretched by up to $80 \%$ strain), which enables the conformability with the human body. The present portable and wearable ISASE demonstrates great potential for cutaneous wound healing, which constitutes an important complement to the current applications in the healthcare field.

\section{Introduction}

The growing interest in advanced stretchable electronics for healthcare applications is evidenced by both the rapid proliferation of research articles in academia and the expanding collection of devices available in consumer markets. ${ }^{[1-4]}$ When integrated with various relevant sensors, these electronics can noninvasively and continuously monitor human vital signs, including body temperature, ${ }^{[5]}$ heart rate, ${ }^{[6]}$ respiration rate, ${ }^{[7]}$ blood pressure, ${ }^{[8]}$ pulse oxygenation, ${ }^{[9]}$ blood glucose, ${ }^{[10]}$ and other physiological information. ${ }^{[11-13]}$ Regular monitoring of vital signs would help to make a real-time assessment of an individual physiological state and to alert the users of certain abnormalities in bodies.

Compared with the prosperity of "passive" monitoring of physiological signs, as illustrated above, there have been rare reports on "active" therapeutic applications of stretchable electronics such as wound healing, which constitutes an
L. Zhang, S. Li, H. Liu, M. Zhang, J. Li, Prof. L. Guo, Prof. Y. Su

State Key Laboratory of Nonlinear Mechanics

Institute of Mechanics

Chinese Academy of Sciences

Beijing 100190, China

E-mail: guoliang@imech.ac.cn; yewangsu@imech.ac.cn

X. Jiang

Key Laboratory of Animal Ecology and Conservation Biology

Institute of Zoology

Chinese Academy of Sciences

Beijing 100101, China

$X$. Jiang

College of Life Science

University of Chinese Academy of Sciences

Beijing 100049, China

The ORCID identification number(s) for the author(s) of this article can be found under https://doi.org/10.1002/admt.201900150.
Prof. W. Jiang, Prof. M. Fang

Institute of Microbiology

Chinese Academy of Sciences

Beijing 100101, China

S. Li, M. Zhang, J. Li, Prof. Y. Su

School of Engineering Science

University of Chinese Academy of Sciences

Beijing 100049, China

Y. Chi, H. Liu, Prof. B. Pan, Prof. Y. Chen

Institute of Solid Mechanics

Beihang University (BUAA)

Beijing 100191, China

Prof. C. Shen

Department of Burn and Plastic Surgery of the Fourth

Medical Center of PLA General Hospital

Beijing 100048, China

DOI: 10.1002/admt.201900150 
attractive complementary direction in the field. There are many effective therapeutic methods for diminishing inflammation, reducing swelling, relieving pain, improving microcirculation, and promoting wound healing, e.g., photodynamic, ${ }^{[14-16]}$ microcurrent, ${ }^{[17-19]}$ and ultrasonic ${ }^{[20-23]}$ treatments, among which the photodynamic therapy has been most widely used due to its noninvasive character and outstanding therapeutic effects. Specifically, the near infrared (NIR) light, used as a low-level laser therapy (LLLT), ${ }^{[24,25]}$ has proven effective in controlling pain and reducing the healing time by stimulating collagen production, fibroblast proliferation, and the local microvasculature as well as by stimulating cellular metabolism to increase the potential for tissue regeneration. ${ }^{[26,27]}$ As a light source of NIR, the light emitting diodes (LEDs) have been recently used for medical treatments, showing great benefits to wound healing. ${ }^{[28-30]}$ With the increasing demand of portable and wearable medical devices for wound healing, the NIR light therapy integrated with stretchable platforms provides an unusual strategy, which may find important applications such as treatments for wounded soldiers, people in travelling, and those at home. Such medical devices are expected to simultaneously achieve the therapy functionalities and flexibility as well as stretchability, thus extending the scope of the stretchable wearable electronic devices.

Here, we introduce a novel infrared skin-like active stretchable electronics (ISASE) for promotion of cutaneous wound healing, which can be conformally mounted anywhere on the human body, including some special parts such as spines and finger/ elbow/knee/ankle joints. By combining stretchable electric circuits with the NIR LED at $850 \mathrm{~nm}$ wavelength, the present ISASE offers a prominent feature that active acceleration of wound healing is fulfilled in a portable and wearable way, thus facilitating the broader use of the photodynamic therapy. With the organic-inorganic composite design of the structure, the device has very high electronic performance, low heat generation, low mechanical stiffness, and high elastic deformability under large strain. The experiments for cure functions show that the ISASE is effective for promoting the migration and proliferation of cells by irradiation of human skin fibroblasts (HSFs) and rat dermal fibroblasts (RDFs), and helps to accelerate the wound healing by stimulating the inflammation inhibition, fibroblast proliferation, collagen production, and local microvasculature establishment. These results demonstrate the great potential of the ISASE for applications in cutaneous wound healing.

Prof. X. Guo, Prof. R. Li

State Key Laboratory of Structural Analysis

for Industrial Equipment

Department of Engineering Mechanics, and International

Research Center for Computational Mechanics

Dalian University of Technology

Dalian 116024, China

E-mail: ruili@dlut.edu.cn

Prof. Y. Su

Beijing Key Laboratory of Engineered Construction

and Mechanobiology

Institute of Mechanics

Chinese Academy of Sciences

Beijing 100190, China

\section{Results and Discussion}

\subsection{Design and Fabrication of ISASE}

The skin-like geometry and stretchability of the ISASE allow its comfortable adhesion to any wound site for therapy. Human skin shows different ranges of strain in various anatomic regions, e.g., $80 \%$ strain for knuckle, $60 \%$ strain for elbow, and $49 \%$ strain for knee. ${ }^{[31]}$ Therefore, large tensile strain is inevitable in the ISASE mounted on the joints to accommodate usual human motions. Figure 1a exhibits the surface strains of a knee and two elbows of a human subject when jogging. Figure $1 \mathrm{~b}$ illustrates the ISASE that is adhered to the knee, with more mounting positions on the human body as shown in Figure S1 in the Supporting Information.

An expanded view of the ISASE structure is shown in Figure 1c, where the device has a multilayer configuration of LEDs/polyimide (PI)/copper (Cu)/PI (1 mm/12.5/18/75 $\mu \mathrm{m}$ thick) from top to bottom, encapsulated by $300 \mu \mathrm{m}$ thick Ecoflex through adhesive elastomer silicone on the front and back sides. A $14 \times 10$ array of LEDs was soldered onto the contact $\mathrm{Cu}$ pads, plus one current-limiting resistance connected in series with each row of 14 LEDs (i.e., there are ten current-limiting resistances in total). The remaining $\mathrm{Cu}$ was coated with the $12.5 \mu \mathrm{m}$ thick PI layer. Details of the configuration and dimensions appear in Figure S2a and Table S1 in the Supporting Information, respectively, with the front and back of the device without capsulation shown in Figure S2b in the Supporting Information. Figure 1d outlines the fabrication process of an ISASE, which is realized by microprocessing strategies, involving etching of $\mathrm{Cu}$ on a PI substrate, lamination of PI on the upside of $\mathrm{Cu}$ sheet, laser cutting for desired configuration of the laminated PI, soldering of LEDs on $\mathrm{Cu}$, and encapsulation of the device. Details appear in the Experimental Section.

\subsection{Medical Mechanism and Mechanical Properties of ISASE}

Nowadays, NIR light with wavelength of 700-1000 nm has been employed for promoting proliferation of many kinds of cells. The underlying medical mechanism of NIR light therapy has been extensively revealed, as shown in Figure S3 in the Supporting Information. Briefly, physiological dose of NIR can activate small GTPase Ras, Raf-1, phosphorylated ERK1/2 pathway. Moreover, NIR has been demonstrated to promote reactive oxygen species (ROS) production through working on mitochondria respiration, another upstream event of phosphorylation of ERK1/2. Recent studies indicated that there were potential light receptors in endoplasmic reticulum (ER). The calcium pool would be triggered when the NIR acts on the ER, and ERK1/2 would then be activated. The phosphorylated ERK1/2 can transport into cell nucleus and interact with different kinds of transcription factors (such as AP1, ATF, EIK-1, and NF-kB), which finally promotes cell proliferation and migration on wound bed. ${ }^{[32-35]}$

To confirm the validity of the ISASE when used in extreme conditions of the human body, we investigate a representative region with large deformation, that is, the elbow. The 

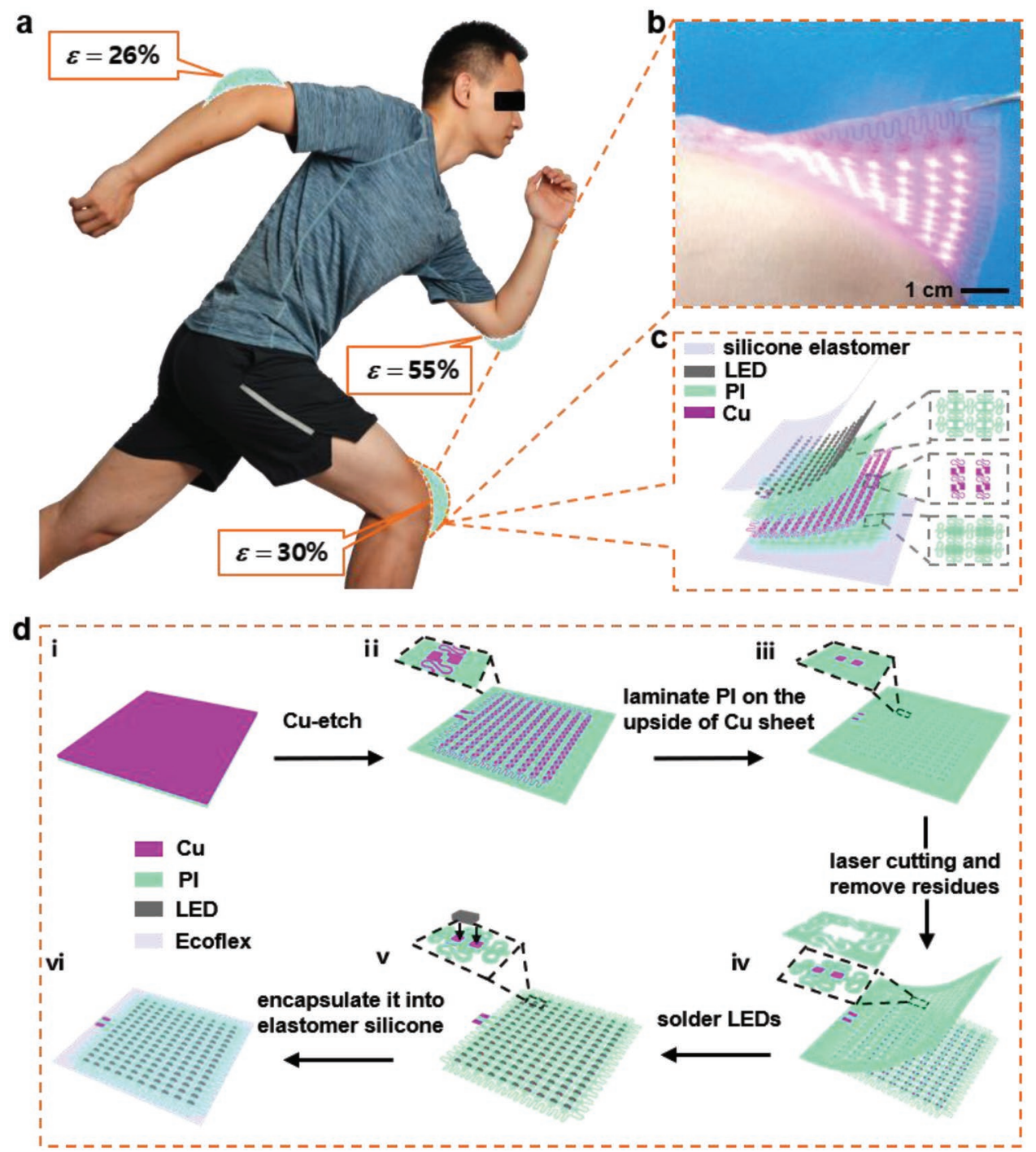

Figure 1. The ISASE for wound healing. a) Different strains of elbows and knee displayed when the human runs. b) Optical images of the lighted device partially lifted up from the skin of the knee. c) Expanded-view schematic illustration of the various constituents of the device, (inset: enlarged view of $\mathrm{Cu}$ and PI layers). d) Fabrication of the ISASE, involving i,ii) etching of $\mathrm{Cu}$ on a PI substrate, iii) lamination of PI on the upside of Cu sheet, iv) laser cutting for desired configuration of the laminated PI, v) soldering of LEDs on $\mathrm{Cu}$, and vi) encapsulation of the device.

stereo-digital image correlation (stereo-DIC) system (Figure S4, Supporting Information) $)^{[36]}$ was used to measure the strain distribution on an elbow (Figure S5, left, Supporting Information), with clear contours of maximum principal strains corresponding to different bending angles of the elbow, as illustrated in Figure S5, right, in the Supporting Information. It is seen that the maximum strain variation of the bent elbow from the initial zero-strain state (with the arm straightened) can reach up to $60 \%$. On the other hand, Figure 2a shows the ISASE that is stretched by up to $70 \%$ strain (see Movie S1 in the Supporting Information), which is remarkably beyond the maximum strain shown in Figure S5 in the Supporting Information; the ISASE can also be folded (Movie S2, Supporting Information) and rolled up (Figure $2 \mathrm{~b}$ ), revealing the capacity of withstanding large deformation. Figure 2c shows the ISASE that works well when stuck on the elbow of an adult human subject, who lifts his forearm and bends the elbow to $70^{\circ}, 90^{\circ}$, and $110^{\circ}$ angles, respectively, while the induced strain increases (see Movies S3 and S4 in the Supporting Information).

The mechanical properties and electrical performances are of primary importance for stretchable electronics such as the ISASE. The uniaxial tensile experiments show that the fracture strain of an $18 \mu \mathrm{m}$ thick monolayer copper is only $1 \%$ but the ductility of the organic-inorganic composite structure with the same $\mathrm{Cu}$ film on a $75 \mu \mathrm{m}$ thick PI substrate can reach up to $17 \%$ (Figure $2 \mathrm{~d}$ ). The mechanism is that a well-bonded substrate carries the film to large tensile strains without rupture by delocalizing deformation in the film; this protective effect of the PI substrate against rupture of the $\mathrm{Cu}$ film is consistent with previous results reported in the literature. ${ }^{[37,38]}$ The finite element analysis (FEA) in Figure 2e confirms that the maximum strain of such a composite structure is far below its ductility when stretched to $60 \%$ applied strain. Figure $2 \mathrm{f}$ shows the uniaxial vertical stretching of the device up to $80 \%$ applied 

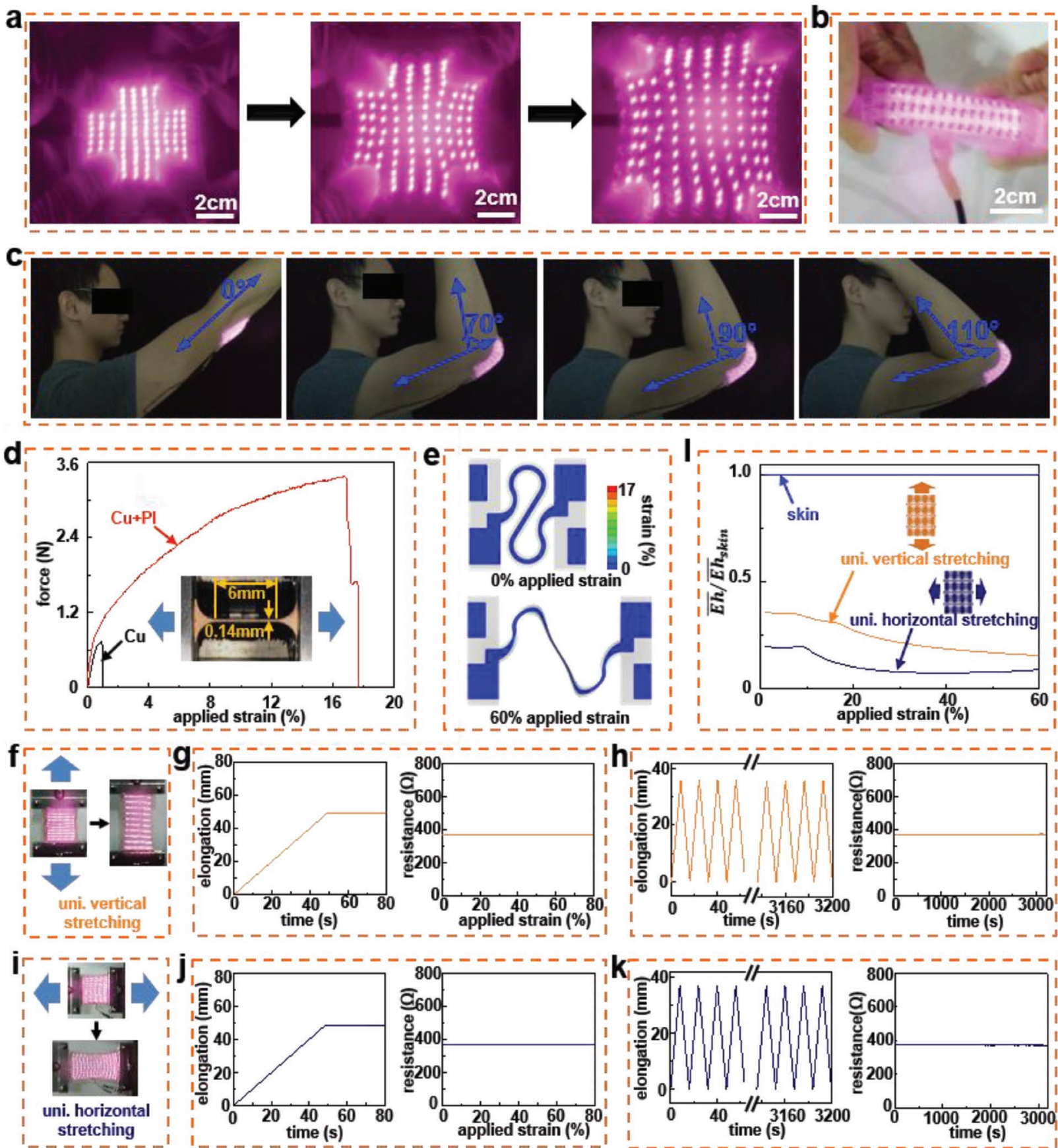

Figure 2. Mechanical properties of the ISASE. Operation of the lighted device when a) biaxially stretched to $70 \%$ strain and b) rolled up. c) An ISASE deformed with elbow movement under four different bending angles: $0^{\circ}, 70^{\circ}, 90^{\circ}$, and $110^{\circ}$. d) Force-applied strain curves of a monolayer $\mathrm{Cu}$ and a $\mathrm{Cu}-\mathrm{PI}$ composite structure under uniaxial tension. e) FEA results of a $\mathrm{Cu}-\mathrm{PI}$ composite interconnect when $0 \%$ and $60 \%$ strains are applied. f) The ISASE under vertical stretching. g) Uniaxial vertical stretching of the device up to $80 \%$ applied strain, with elongation versus time (left) and electrical resistance versus applied strain (right) plotted. h) Elongation and electrical resistance of the device under 200 cycles of vertical tensile loading, with $60 \%$ maximum strain. i) The ISASE under horizontal stretching. j) Uniaxial horizontal stretching of the device up to $80 \%$ applied strain, with elongation versus time (left) and electrical resistance versus applied strain (right) plotted. k) Elongation and electrical resistance of the device under 200 cycles of horizontal tensile loading, with $60 \%$ maximum strain. I) Relative tensile stiffnesses of the device to human skin in uniaxial vertical and horizontal stretching.

strain, with the elongation plotted versus time in the left frame of Figure $2 \mathrm{~g}$, the right frame of which shows that the electrical resistance of the device keeps unchanged during stretching and the brightness of the LEDs is stable.
To study the wear resistance, the ISASE under cyclic loading conditions is considered. The results indicate that the electrical resistance remains stable after 200 cycles of tensile loading, with $60 \%$ maximum strain (Figure $2 \mathrm{~h}$ ), and the LEDs work stably. 
In the same manner as the case of vertical stretching, both the uniaxial horizontal stretching (Figure 2i) and cyclic loading test are conducted. The results are shown in Figure $2 \mathrm{j}, \mathrm{k}$, corresponding to Figure $2 \mathrm{~g}$, h for the case of vertical loading. It is found that, for $80 \%$ applied strain (Figure 2j) and 200 cycles of tensile loading with $60 \%$ maximum strain (Figure $2 \mathrm{k}$ ), the electrical resistance and brightness of the LEDs remain unchanged, indicating the robustness of the present design. Figure 21 plots the relative tensile stiffnesses of the device to human skin (with Young's modulus $400 \mathrm{kPa}$ and thickness $1 \mathrm{~mm})^{[39]}$ in uniaxial vertical and horizontal stretching, which indicates that the stiffnesses of the device in both directions are far below that of skin, highlighting the excellent mechanical properties of the present composite structure.

The optical characteristics of the ISASE are also measured and calculated (Figure S6, Supporting Information). When the input current of the infrared arrays is $200 \mathrm{~mA}$, the passing current in each LED is $20 \mathrm{~mA}$, and the measured radiation intensity of each LED is $83 \mathrm{~mW} \mathrm{sr}{ }^{-1}$. When the irradiation distance is $1.5 \mathrm{~cm}$, the intensity distribution of the ISASE on the receiving surface is calculated using MAPLE software package (Note S1, Supporting Information). The peak value of radiation intensity calculated is $11.34 \mathrm{~mW} \mathrm{~cm}^{-2}$; at least $90 \%$ intensity is realized in the central $2 \times 2 \mathrm{~cm}^{2}$ region and $80 \%$ in the central $3 \times 3 \mathrm{~cm}^{2}$ region of the ISASE, indicating a uniform radiation distributed in a large region of the device.

Overall, compared with most previous stretchable electronics that have monolayer inorganic interconnects, the organicinorganic composite design of the structure is developed in the present ISASE, by which very low stiffness, high elastic deformability, and excellent insulation/protection of the device has been realized. On the other hand, the novel prestrain-free design yields relatively thick interconnect layout (with thickness of tens of micrometers) compared with previously used ultrathin structures (involving metals, insulators, or semiconductors with thicknesses typically between $\approx 100 \mathrm{~nm}$ and $\approx 1 \mu \mathrm{m}$ ). This feature highlights the exceptional advantage that very low electrical resistance and highly efficient heat dissipation in interconnects is achieved for enhanced operation of arrays of high-power LEDs in the ISASE. ${ }^{[4]}$

\subsection{Skin Cell Proliferation and Migration by ISASE}

It is well known that low power infrared irradiation can promote cell proliferation while high intensity illumination induces apoptosis. ${ }^{[40,41]}$ To select an appropriate dose of NIR radiation for promoting skin cell proliferation, HSFs and RDFs grown in 96-well plates were treated with a series of input electric currents, i.e., 100, 150, 200, and $250 \mathrm{~mA}$, for $20 \mathrm{~min}$ after $24 \mathrm{~h}$ starvation, which correspond to radiation powers of 7.20, 9.30, 11.22 , and $12.70 \mathrm{~mW} \mathrm{~cm}{ }^{-2}$, respectively (Figure 3a). The lighted ISASE at different input currents are shown in Figure S7 in the Supporting Information, with the corresponding data shown in Table S2 in the Supporting Information. The cell counts were performed using MTT at $24 \mathrm{~h}$ following irradiation. The result shows that the cells increase in a dose-dependent manner: 200 and $250 \mathrm{~mA}$ for $20 \mathrm{~min}$ significantly accelerate the proliferation of both HSFs (Figure 3b) and RDFs (Figure 3c), but neither
50 nor $300 \mathrm{~mA}$ has significant effects on the proliferation (data not shown). Simultaneously, we kept the same dose for further experiments and found that the phosphorylation of ERK, as the essential course for initiating cell proliferation, was dramatically increased in 6-well plate cultured HSFs (Figure 3d) and RDFs (Figure 3e) after NIR irradiation. The following cell cycle analyzed via flow cytometry confirmed that the dose of $13.46 \mathrm{~J} \mathrm{~cm}^{-2}$ with $200 \mathrm{~mA}$ input electric current of the device could effectively accelerate repopulation of skin cells, as indicated by comparison between the cell counts before and after NIR irradiation (Figure 3f,g). Fibroblasts migration is a key coevent during wound healing and is also under ERK pathway regulation. ${ }^{[42]}$ To verify this, the cell scratch assay was conducted with 6-well plate cultured cells. The scratches were fabricated via pipette tips and then treated with/without infrared array irradiation for $20 \mathrm{~min}$ at the dose of $13.46 \mathrm{~J} \mathrm{~cm}^{-2}$. The 6 -well plate was surveyed under a microscope $24 \mathrm{~h}$ later, showing that many more HSFs and RDFs migrated to the blank area caused by scratch after infrared array treatment compared with the nontreated group, as indicated in Figure 3h,i that was a set extracted from three independent experiments showing similar results.

\subsection{Wound Closure in Rat by ISASE}

The NIR light with 700-1000 nm wavelength has been used in clinical treatment for promoting wound healing and many previous studies showed that the NIR light can accelerate wound healing through inflammation inhibition, angiogenesis enhancement, and collagen deposition. ${ }^{[43-46]}$ Here, we aim to demonstrate whether the ISASE equipped with NIR LEDs could accelerate skin wound healing of rats. Figure 4a shows the wound modeling that is established on the back of a rat. The ISASE was overlaid on the wound once a day at an efficient irradiation dose of $13.46 \mathrm{~J} \mathrm{~cm}^{-2}$ for skin cell since the second day of trauma (Figure S8, Supporting Information). In order to keep the rat quiet, we put it into a transparent plastic bottle, and cut a circular opening above the wound. The nontreated wounded rats served as control. The wound sizes were observed and recorded under the same scale plate before each irradiation. It is obvious that NIR light irradiation significantly accelerates the wound healing and shortens the cure time (when over $95 \%$ of wound closure is observed) ${ }^{[47}$ by at least two days (Figure 4b,c), as reflected in Figure 4c by less than ten days for wound closure with NIR light irradiation versus 12 days without irradiation.

In the course of irradiation, the treated rats showed less weeping and suppurating, indicating the inhibited inflammatory response over the wound bed. To identify the putative appearance, hematoxylin and eosin (H\&E) stain was performed to measure the inflammation cells in skin tissue over wounds. ${ }^{[45,48]}$ On day 3, the regenerated tissue was abnormal, with much more granulation tissue, in the nonirradiated control group (Figure 4d-i) compared with the irradiated rat (Figure 4d-ii). More importantly, the severe inflammation in the wound bed was efficiently inhibited via NIR light irradiation. At the end of the observation period, we found that there was still moderate inflammation in control rats (Figure 4d-iii) compared with the irradiated rats (Figure 4d-iv). 

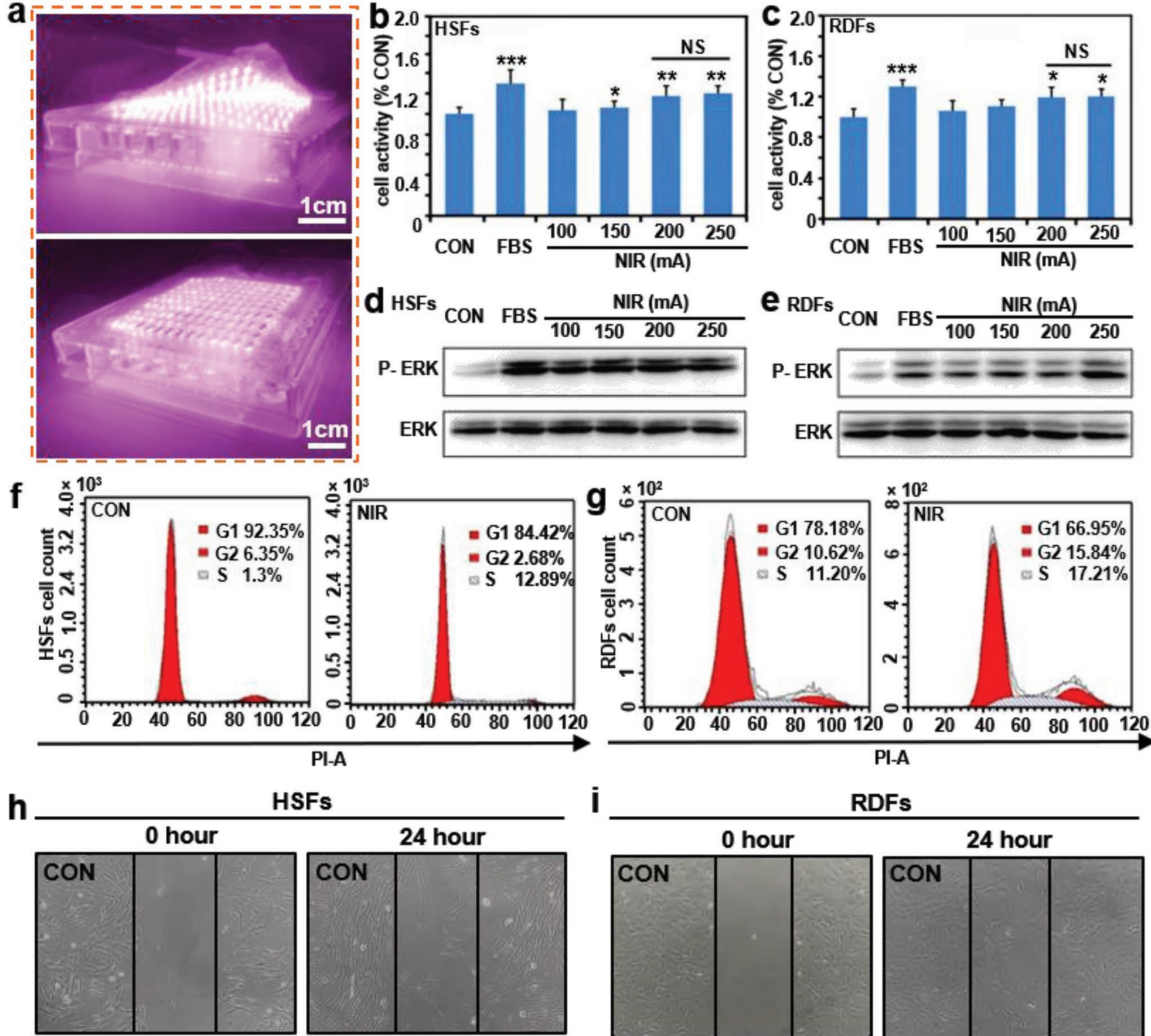

HSFs

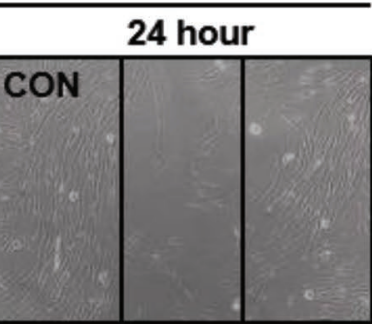

i

PI-A
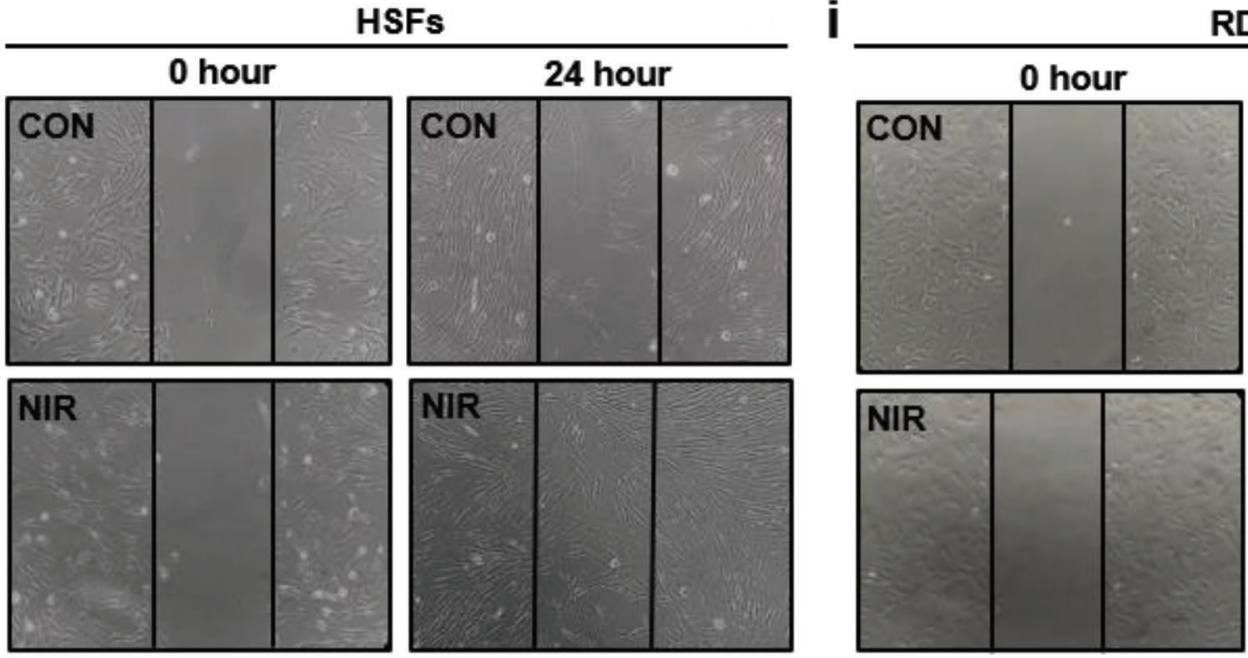

RDFs
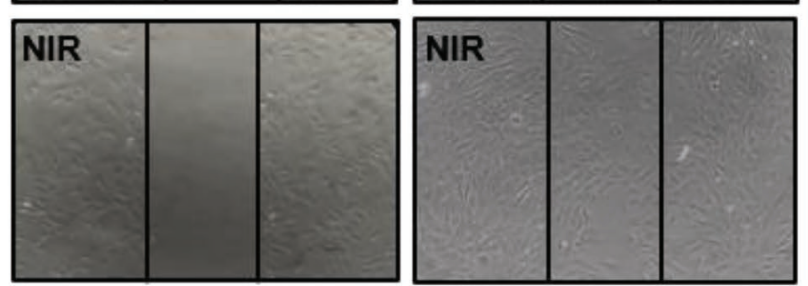

Figure 3. Skin cell proliferation and migration by the ISASE. a) Operational flowchart of ISASE irradiation on 96-well plate. b) HSF cells and c) rat dermal fibroblasts (RDFs) were seeded on 96-well microplates for $12 \mathrm{~h}$ flowed with $24 \mathrm{~h}$ serum deprivation and then irradiated with the ISASE at exhibited input current for 20 min, respectively, or treated with 10\% FBS. Cell counts were assessed by the MTT assay after $24 \mathrm{~h}$. Data represented as mean \pm SEM ( $n=10 ; * P<0.05, * * P<0.01$, $* * * P<0.001$ and vs control cells). d) HSF cells and e) RDFs seeded on 6-well microplates were treated and then lysed for western blot assay to analyze the ERK phosphorylation level. f,g) Flow cytometry analysis of HSF cell and rat primary skin cell cycle under mentioned treatment. Photomicrographs for migration of $h$ ) HSF cells and i) RDFs following a scratch wound.

Extracellular matrix (ECM) is mainly composed of collagen particles, which constitutes over $70 \%$ of the skin. Collagen deposition is thus the key for repair and regeneration of the skin. ${ }^{[49]}$ Therefore, Masson's trichrome stain was performed to explore whether ISASE irradiation can promote collagen deposition. ${ }^{[45]}$ The results showed that collagen particles were orderly restructured on day 3 in treated rats (Figure 4e-ii), but it was substituted with edematous fluid in control rats (Figure 4e-i). 
a
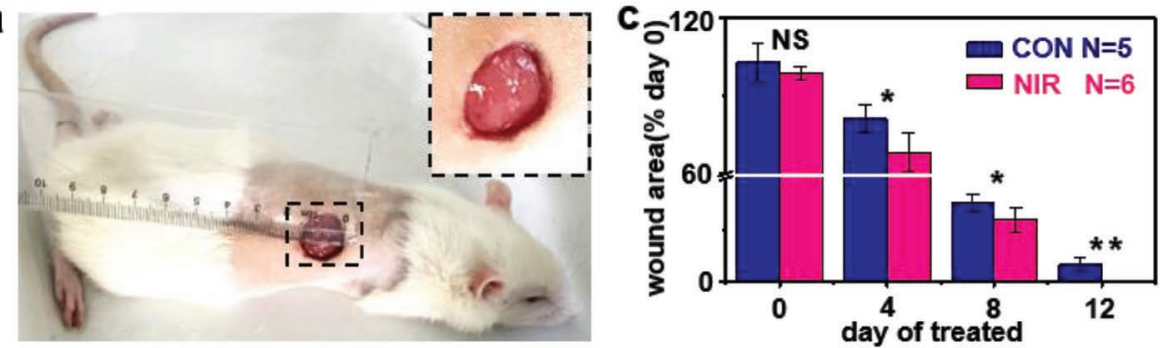

b
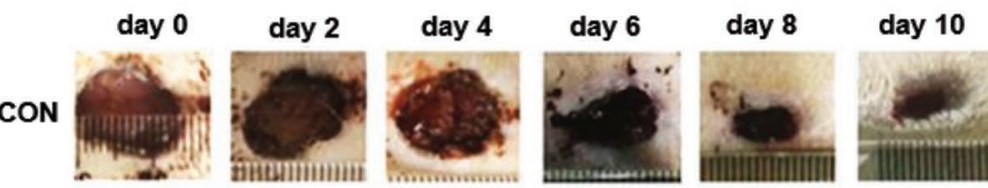

day 12

NIR
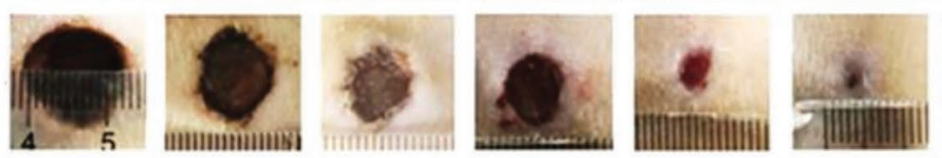

monmmoming
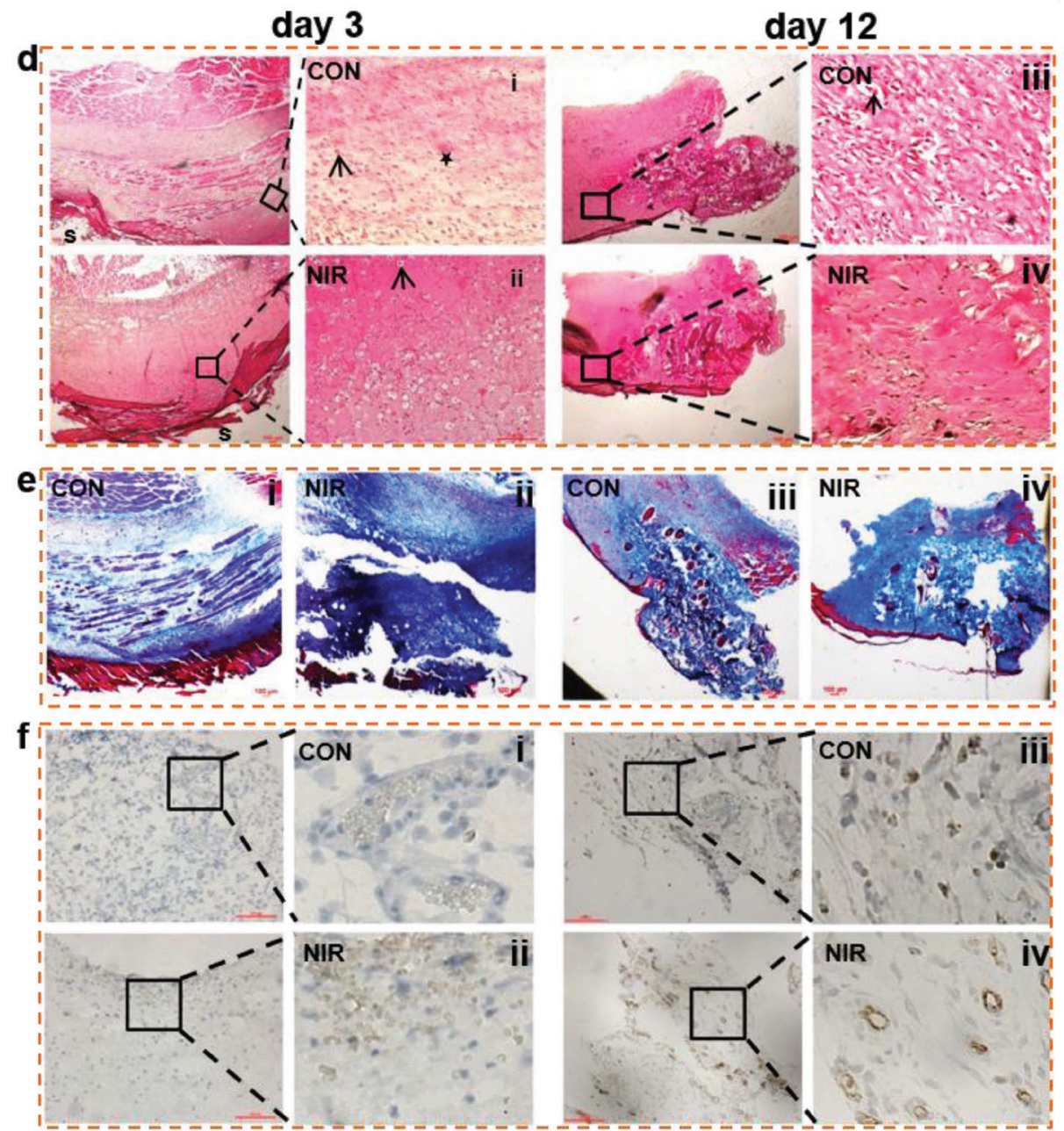

Figure 4. Wound closure in rat by the ISASE and histopathological analyses of wound healing. a) Photograph of wound modeling on back of rat. b) Typical photographs of wound beds for control and irradiation groups. c) Percentage of original wound area at $0,4,8$, and 12 days after wounding. Data are represented as mean $\pm \operatorname{SEM}(n=10 ; * P<0.05, * * P<0.01$ and vs control cells). d) Histological section of a healed wound on day 3 and day 12 after surgery (H\&E staining). s: scab; arrows: inflammatory cells; asterisk: granulation tissue. e) Masson's trichrome staining for the deposited collagen (blue) on section of healed wounds on day 3 and day 12 after surgery (Masson's trichrome stain). Scale bar: $100 \mu \mathrm{m}$. $\mathrm{f}$ ) Typical photographs of CD31 staining on day 3 and day 12 of surgery. Scale bar: $100 \mu \mathrm{m}$. 
The Masson's trichrome staining revealed that there was a significantly higher collagen deposition in treated rats than in control rats on day 12 postwounding (Figure 4e-iii,iv).

To further confirm the good effects of ISASE irradiation on the promotion of wound restoration, capillaries in the newborn tissue was stained for CD31. ${ }^{[50]}$ On day 3 of wound healing, unassembled capillary cells were more significant in irradiated rats compared with control rats (Figure 4f-i,ii). On day 12, irradiated rats continued to demonstrate much more mature capillary cells compared with control rats (Figure 4 f-iii,iv). These results illustrate that ISASE irradiation is an effective approach to enhancing angiogenesis in skin wound healing.

\section{Conclusions}

In this study, we introduce the novel infrared skin-like active stretchable electronics that can effectively promote cutaneous wound healing. The present device is realized by using an unusual strategy that integrates the NIR light therapy with flexible and stretchable platforms, thus achieving very low stiffness and high elastic deformability under large strain; this guarantees the conformability with the human body toward portable and wearable healing. Due to the relatively thick interconnect layout, the present structural design provides an exceptional advantage that very low resistance is realized for enhanced operation of arrays of high-power LEDs by reducing harmful heat generation. The experiments of cells in vitro and cutaneous wound healing in vivo confirm that the ISASE is feasible for promoting the migration and proliferation of cells by irradiation of human skin fibroblasts and rat dermal fibroblasts, and is effective for accelerating the wound healing accompanied by inflammation inhibition, fibroblast proliferation, collagen production, and local microvasculature establishment. Specifically, compared with the normal treatment for a wounded rat with the cure time of 12 days, the use of ISASE significantly shortens the time by at least two days. The comprehensive design, fabrication, and performance characterization of the ISASE highlight the great potential for stretchable, wearable, and portable electronics with active therapeutic applications toward wound healing of human beings. While a general beneficial effect on overall healing has been shown, more specific analysis of the cellular responses and mechanisms involved will be further explored in future studies.

\section{Experimental Section}

Fabrication Process of ISASE: The circuit of the ISASE was designed to be series-parallel connections, with 14 LEDs and one currentlimiting resistance of type 0603 (length: $1.6 \mathrm{~mm}$, width: $0.8 \mathrm{~mm}$, and height: $0.7 \mathrm{~mm}$ ) connected in series as a row, and ten such rows were in parallel. The device was fabricated starting from the substrate that was made from flexible single-sided Cu-clad laminate consisting of $18 \mu \mathrm{m}$ thick $\mathrm{Cu}$ sheet and $75 \mu \mathrm{m}$ thick supporting PI (Figure 1d-i). The fabrication began with patterning $140 \mu \mathrm{m}$ wide $\mathrm{Cu}$ meanders and island-like bonding pads by photolithography and wet etching along with the standard design (Figure 1d-ii). A $12.5 \mu \mathrm{m}$ thick layer of PI that was hollowed out in the location corresponding to the bonding pads was coated on the upside of Cu meanders (Figure 1d-iii). The island bridgelike outline was structured in one step by laser cutting, and then the flexible printed circuit board (FPCB) residues were removed by peeling them off (Figure 1d-iv). The width of two layers of PI was $340 \mu \mathrm{m}$ in stretchable interconnects, which was wider than $\mathrm{Cu}$ meanders to protect them against damage under stretch. The substrate fabrication was finalized by assembling the infrared LEDs of type 0805 (length: $2.0 \mathrm{~mm}$, width: $1.25 \mathrm{~mm}$, and height: $1.0 \mathrm{~mm}$ ) with peak wavelength of $850 \mathrm{~nm}$ on the "island" (Figure 1d-v). The entire stretchable circuit was finally encapsulated by $300 \mu \mathrm{m}$ thick Ecoflex films through adhesive elastomer silicone on the front and back sides (Figure 1d-vi; Note S2, Supporting Information).

Tensile Experiments of $\mathrm{Cu}$ and $\mathrm{Cu}-\mathrm{PI}$ Composite Structure: The samples were cut into dog-bone shape by laser (Figure 2d). Sample 1 was cut from a thin $\mathrm{Cu}$ film and sample 2 from a single-sided Cu-clad laminate with a PI substrate. The thickness and width of the samples were the same as the meanders in ISASE; the length was $6 \mathrm{~mm}$. The uniaxial tensile tests of the samples were performed with a stretcher with $0.1 \mathrm{~mm} \mathrm{~s}^{-1}$ loading rate.

Finite Element Modeling: The FEA was conducted in Abaqus, a widely accepted commercial software. PI and $\mathrm{Cu}$ were treated as linear elastic and ideal elastic plastic materials, respectively. The Young's modulus and Poisson's ratio of PI are $2.5 \mathrm{GPa}$ and 0.34 , respectively. The Young's modulus, Poisson's ratio, and yield stress of $\mathrm{Cu}$ are $124 \mathrm{GPa}, 0.34$, and $372 \mathrm{MPa}$, respectively. The shell element S4R was adopted for both PI and $\mathrm{Cu}$.

Tensile Experiment of ISASE: The tensile test of the ISASE was performed with a fatigue tester. Under a constant current source, a multimeter was used to monitor the voltage variation and to achieve automatic data collection. The dynamic resistance of the device was obtained by the voltage divided by the current. The test included uniaxial stretching and cyclic loading in both vertical and horizontal directions. Up to $80 \%$ strain was applied in uniaxial stretching with $1 \mathrm{~mm} \mathrm{~s}$ loading rate. For cyclic test, 200 cycles of tensile loading with $60 \%$ maximum strain were applied, with a period of $16 \mathrm{~s}$.

Animals: Pregnant SD rats and adult male SD rats were purchased from Vital River Laboratory Animal Technology (Beijing, China). All animal studies were approved by the Institutional Animal Care and Use Committee of Institute of Zoology (Chinese Academy of Sciences).

Rat Dermal Fibroblasts Isolation and Cell Culture::[1] $1 \times 1 \mathrm{~cm}^{2}$ skin was removed from a neonatal rat, then rinsed with PBS solution for three times, and cut into $5 \times 5 \mathrm{~mm}^{2}$ pieces. The tissue sections were digested with $0.25 \%$ trypsin at $37^{\circ} \mathrm{C}$ for $30 \mathrm{~min}$, and then the epidermis, subcutaneous muscle, and adipose tissues were removed with tweezers, retaining the dermis only. The skin tissue samples were sectioned and digested with $1 \%$ type I collagenase at $37^{\circ} \mathrm{C}$ for $1 \mathrm{~h}$. The mixture was filtered with 200 mesh sieves and centrifugated at $1000 \mathrm{rpm}$ at room temperature for $15 \mathrm{~min}$, and the deposited cells were collected. The isolated cells were cultured in DMEM medium with $10 \%$ fetal bovine serum (FBS, Gibco; Thermo Fisher Scientific Inc.), maintained at $37{ }^{\circ} \mathrm{C}$ and $5 \% \mathrm{CO}_{2}$. Cell passage cultivation was conducted every three days for $1 \mathrm{cycle}$, and then nonobjective cells died out and remaining dermal fibroblasts were prepared for the following experiment.

ISASE Irradiation on Cells: Device assembled with fabrication of the stretchable circuit configuration and infrared LEDs that emit peak wavelength of $850 \mathrm{~nm}$ was used as the ISASE. The irradiation experiment was conducted as described previously. ${ }^{[2]}$ Briefly, the ISASE was located on the plate cap at a short distance to minimize heat interfering and an infrared detector was used to blank the energy loss resulting from plate, medium, and distance. To get the efficient dose, cells were seeded on plates in DMEM medium with $10 \%$ FBS. The cell was changed with FBS-deprived DMEM for $24 \mathrm{~h}$ when plate was $70 \%$ covered, then irradiated with the device at 50,100, 150,200, 250, and $300 \mathrm{~mA}$ input current for $20 \mathrm{~min}$, and cultured for another $24 \mathrm{~h}$ for MTT, western blot, or flow cytometry assays. The cells without irradiation were covered with light-tight tin foil sheet.

Scratch Wound Healing Assays: For the cell migration assays, cells were seeded on 6-well plate in DMEM medium with 10\% FBS. The scratch was conducted with $200 \mu \mathrm{L}$ yellow beveled tips and FBS-deprived DMEM was changed when the plate was $70 \%$ covered, then irradiated 
with the ISASE at total dose of $13.46 \mathrm{~J} \mathrm{~cm}^{-2}$ for $20 \mathrm{~min}$, and cultured for another $24 \mathrm{~h}$. The migrated cells were observed with microscope in starch area.

Laboratory Animals and Wound Model Creating: Adult male SD rats with 200-220 g body weight were divided into three groups and housed separately with one rat per cage. For the wound model, rats were anaesthetized by intraperitoneal injection of avertin to create wounds. The skin was clipped and disinfected with $75 \%$ alcohol. $1.5 \mathrm{~cm}$ of skin uniform in diameter was removed from the area of dorsal part of the rats with the aid of a round seal. The entire wound was left open, and the wound area on day 0 was measured on the second postsurgery wound day. The rats were irradiated by 12 sessions, accepted the dose of $13.46 \mathrm{~J} \mathrm{~cm}^{-2}$ in $20 \mathrm{~min}$ for one time per day, and $24 \mathrm{~h}$ after the induction of the wound. A transparent plastic bottle was used as a fixing holder to keep the animal stable during the ISASE irradiation. Due to the wound area being much less than the coverage area of device, a $1.5 \mathrm{~mm}$ thick black Ecoflex (Note S3, Supporting Information) coating was prepared on the infrared LEDs array to block the infrared light in addition to the wound area (Figure S8, Supporting Information).

Estimation of the Rate of Wound Closure: The wound closure areas each day were assessed via CAD software. The reduced wound area of day 0 divided by day 0 wound areas was defined as wound closure rate. The wound closure rates of continually 12 days were made into wound healing curve.

Informed signed consent was obtained from the subjects involved in this study.

\section{Supporting Information}

Supporting Information is available from the Wiley Online Library or from the author.

\section{Acknowledgements}

L.Z. and X.J. contributed equally to this work. The authors gratefully acknowledge the support from the National Natural Science Foundation of China (Grants 11772331, 11572323, and 11302038). Y.S. acknowledges the support from Chinese Academy of Sciences via the "Hundred Talent Program," Strategic Priority Research Program of the Chinese Academy of Sciences (No. XDB22040501). R.L. acknowledges the support from the Young Elite Scientists Sponsorship Program by CAST (No. 2015QNRC001), Opening Fund of State Key Laboratory of Nonlinear Mechanics, Chinese Academy of Sciences, and Fundamental Research Funds for the Central Universities of China (No. DUT18GF101). X.G. acknowledges the support from the National Key Research and Development Plan (Grant Nos. 2016YFB0201600, 2016YFB0201601, 2017YFB0202800, and 2017YFB0202802), Program for Changjiang Scholars, Innovative Research Team in University (PCSIRT), and 111 Project (Grant No. B14013).

\section{Conflict of Interest}

The authors declare no conflict of interest.

\section{Keywords}

cutaneous wound healing, fibroblast proliferation, inflammation inhibition, near infrared therapy, stretchable electronics

Received: February 18, 2019

Revised: March 27, 2019

Published online: May 14, 2019
[1] J. Heikenfeld, A. Jajack, J. Rogers, P. Gutruf, L. Tian, T. Pan, R. Li, M. Khine, J. Kim, J. Wang, J. Kim, Lab Chip 2018, 18, 217.

[2] J. Kim, G. A. Salvatore, H. Araki, A. M. Chiarelli, Z. Xie, A. Banks, X. Sheng, Y. Liu, J. W. Lee, K. I. Jang, S. Y. Heo, K. Cho, H. Luo, B. Zimmerman, J. Kim, L. Yan, X. Feng, S. Xu, M. Fabiani, G. Gratton, Y. Huang, U. Paik, J. A. Rogers, Sci. Adv. 2016, 2, e1600418.

[3] T. Yokota, P. Zalar, M. Kaltenbrunner, H. Jinno, N. Matsuhisa, H. Kitanosako, Y. Tachibana, W. Yukita, M. Koizumi, T. Someya, Sci. Adv. 2016, 2, e1501856.

[4] Y. Su, X. Ping, K. J. Yu, J. W. Lee, J. A. Fan, B. Wang, M. Li, R. Li, D. V. Harburg, Y. Huang, C. Yu, S. Mao, J. Shim, Q. Yang, P. Y. Lee, A. Armonas, K. J. Choi, Y. Yang, U. Paik, T. Chang, T. J. Dawidczyk, Y. Huang, S. Wang, J. A. Rogers, Adv. Mater. 2017, 29, 1604989.

[5] Y. Hattori, L. Falgout, W. Lee, S. Y. Jung, E. Poon, J. W. Lee, I. Na, A. Geisler, D. Sadhwani, Y. Zhang, Y. Su, X. Wang, Z. Liu, J. Xia, H. Cheng, R. C. Webb, A. P. Bonifas, P. Won, J. W. Jeong, K. I. Jang, Y. M. Song, B. Nardone, M. Nodzenski, J. A. Fan, Y. Huang, D. P. West, A. S. Paller, M. Alam, W. H. Yeo, J. A. Rogers, Adv. Healthcare Mater. 2014, 3, 1597.

[6] Y. Khan, M. Garg, Q. Gui, M. Schadt, A. Gaikwad, D. Han, N. A. D. Yamamoto, P. Hart, R. Welte, W. Wilson, S. Czarnecki, M. Poliks, Z. Jin, K. Ghose, F. Egitto, J. Turner, A. C. Arias, Adv. Funct. Mater. 2016, 26, 8764.

[7] C. Mao, H. Zhang, Z. Lu, Smart Mater. Struct. 2017, 26, 095033.

[8] M. K. Choi, O. K. Park, C. Choi, S. Qiao, R. Ghaffari, J. Kim, D. J. Lee, M. Kim, W. Hyun, S. J. Kim, H. J. Hwang, S. H. Kwon, T. Hyeon, N. Lu, D. H. Kim, Adv. Healthcare Mater. 2016, 5, 80.

[9] T. Lu, E. J. Markvicka, Y. Jin, C. Majidi, ACS Appl. Mater. Interfaces 2017, 9, 22055.

[10] W. Gao, S. Emaminejad, H. Y. Y. Nyein, S. Challa, K. Chen, A. Peck, H. M. Fahad, H. Ota, H. Shiraki, D. Kiriya, D. H. Lien, G. A. Brooks, R. W. Davis, A. Javey, Nature 2016, 529, 509.

[11] A. Koh, D. Kang, Y. Xue, S. Lee, R. M. Pielak, J. Kim, T. Hwang, S. Min, A. Banks, P. Bastien, M. C. Manco, L. Wang, K. R. Ammann, K. I. Jang, P. Won, S. Han, R. Ghaffari, U. Paik, M. J. Slepian, G. Balooch, Y. Huang, J. A. Rogers, Sci. Transl. Med. 2016, 8, 366 ral65.

[12] H. Y. Nyein, W. Gao, Z. Shahpar, S. Emaminejad, S. Challa, K. Chen, H. M. Fahad, L. C. Tai, H. Ota, R. W. Davis, A. Javey, ACS Nano 2016, 10, 7216.

[13] R. C. Webb, Y. Ma, S. Krishnan, Y. Li, S. Yoon, X. Guo, X. Feng, Y. Shi, M. Seidel, N. H. Cho, Sci. Adv. 2015, 1, e1500701.

[14] M. W. Cheon, T. G. Kim, Y. S. Lee, S. H. Kim, Pers. Ubiquitous Comput. 2013, 17, 1421.

[15] H. Chung, T. Dai, S. K. Sharma, Y. Y. Huang, J. D. Carroll, M. R. Hamblin, Ann. Biomed. Eng. 2012, 40, 516.

[16] S. C. Nunez, C. M. Franca, D. F. Silva, G. E. Nogueira, R. A. Prates, M. S. Ribeiro, Lasers Med. Sci. 2013, 28, 633.

[17] L. Poltawski, M. Johnson, T. Watson, Physiother. Res. Int. 2012, 17, 157.

[18] L. C. Kloth, in Wound Healing: Electrical Stimulation (Eds: A. F. Falabella, R. S. Kirsner), Taylor \& Francis, NY 2005, pp. 439-479.

[19] A. J. Robinson, in Clinical Electrophysiology: Electrotherapy and Electrophysical Testing, 3rd ed. (Eds: A. J. Robinson, M. L. Snyder), Lippincott Williams \& Wilkins, Philadelphia 2008, pp. 275-299.

[20] K. Osumi, S. Matsuda, N. Fujimura, K. Matsubara, M. Kitago, O. Itano, C. Ogino, N. Shimizu, H. Obara, Y. Kitagawa, J. Biomed. Mater. Res., Part B 2017, 105, 2344.

[21] S. L. Jeremias Júnior, G. L. Camanho, A. C. Bassit, A. Forgas, S. J. Ingham, R. J. Abdalla, J. Orthop. Sports Phys. Ther. 2011, 41, 526.

[22] C. S. Enwemeka, Am. J. Phys. Med. Rehabil. 1989, 68, 283.

[23] E. A. Abdulhameed, H. H. Enezei, M. Omar, A. Komori, Y. Sugita, F. A. Hegazy, S. Ar, H. Maeda, M. K. Alam, J. Hard Tissue Biol. 2017, 26, 323. 
[24] N. T. D. Sousa, R. C. Gomes, M. F. Santos, H. E. Brandino, R. Martinez, R. R. D. J. Guirro, Lasers Med. Sci. 2016, 31, 549.

[25] P. Brassolatti, P. S. Bossini, M. C. Oliveira, H. W. Kido, C. R. Tim L. A. Lopes, L. R. D. Avo, F. M. A. Moreira, N. A. Parizotto, Microsc Res. Tech. 2016, 79, 313.

[26] P. C. Silveira, K. B. Ferreira, F. R. D. Rocha, B. L. Pieri, G. S. Pedroso, C. T. D. Souza, R. T. Nesi, R. A. Pinho, Inflammation 2016, 39, 1395.

[27] M. H. D. V. Catao, C. F. Nonaka, R. L. de Albuquerque Jr., P. M. Bento, R. de Oliveira Costa, Lasers Med. Sci. 2015, 30, 421.

[28] G. Y. Lee, W. S. Kim, J. Cosmet. Laser Ther. 2012, 14, 107.

[29] M. A. Trelles, I. Allones, E. Mayo, Med. Laser Appl. 2006, 21, 165.

[30] P. Wendling, Skin Allergy News 2005, 36, 32.

[31] R. Wang, N. Jiang, J. Su, Q. Yin, Y. Zhang, Z. Liu, H. Lin F. A. Moura, N. Yuan, S. Roth, R. S. Rome, R. O. Robles, K. Inoue, S. Yin, S. Fang, W. Wang, J. Ding, L. Shi, R. H. Baughman, Z. Liu, Adv. Funct. Mater. 2017, 27, 1702134.

[32] H. Gunji, R. Kunimatsu, Y. Tsuka, Y. Yoshimi, K. Sumi, T. Awada, K. Nakajima, A. Kimura, T. Hiraki, N. Hirose, Lasers Surg. Med. 2018, 50, 772 .

[33] M. Migliario, M. Sabbatini, C. Mortellaro, F. Reno, J. Biophotonics 2018, 11, e201800025.

[34] X. Gao, D. Xing, J. Biomed. Sci. 2009, 16, 4.

[35] X. Gao, D. Xing, L. Liu, Y. Tang, J. Cell. Physiol. 2009, 219, 535.

[36] Y. Chi, L. Yu, B. Pan, Opt. Lasers Eng. 2017, 000, 1.

[37] Y. Xiang, T. Li, Z. Suo, J. J. Vlassak, Appl. Phys. Lett. 2005, 87, 161910.

[38] T. Li, Z. Huang, Z. Suo, S. P. Lacour, S. Wagner, Appl. Phys. Lett 2004, 85, 3435.
[39] D. H. Kim, N. Lu, R. Ma, Y. S. Kim, R. H. Kim, S. Wang, J. Wu, S. M. Won, H. Tao, A. Islam, K. J. Yu, T. I. Kim, R. Chowdhury, M. Ying, L. Xu, M. Li, H. J. Chung, H. Keum, M. McCormick, P. Liu, Y. W. Zhang, F. G. Omenetto, Y. Huang, T. Coleman, J. A. Rogers, Science 2011, 333, 838.

[40] J. Feng, Y. Zhang, D. Xing, Cell. Signalling 2012, 24, 1116.

[41] S. Wu, F. Zhou, Y. Wei, W. R. Chen, Q. Chen, D. Xing, Antioxid. Redox Signaling 2014, 20, 733.

[42] A. J. Singer, R. A. Clark, N. Engl. J. Med. 1999, 341, 738.

[43] Y. Qiao, F. Ma, C. Liu, B. Zhou, Q. Wei, W. Li, D. Zhong, Y. Li, M. Zhou, ACS Appl. Mater. Interfaces 2017, 10, 193.

[44] A. Yadav, A. Gupta, Photodermatol., Photoimmunol. Photomed. 2017, 33, 4.

[45] W. P. Li, C. H. Su, S. J. Wang, F. J. Tsai, C. T. Chang, M. C. Liao, C. C. Yu, T. T. Vi Tran, C. N. Lee, W. T. Chiu, ACS Nano 2017, 11, 5826.

[46] X. Xu, X. Liu, T. Lei, Z. Cui, X. Yang, S. Zhu, Z. Li, X. Yuan, Y. Zheng, K. W. K. Yeung, Acta Biomater. 2018, 77, 352.

[47] M. Ackermann, T. Wolloscheck, A. Wellmann, V. W. Li, W. W. Li, M. A. Konerding, J. Mol. Med. 2011, 27, 647.

[48] A. N. Otterço, P. Brassolatti, A. L. M. Andrade, L. R. S. Avó, P. S. Bossini, N. A. E. Parizotto, Lasers Med. Sci. 2018, 33, 1867.

[49] P. S. Briquez, J. A. Hubbell, M. M. Martino, Adv. Wound Care 2015, 4,479 .

[50] M. Castela, D. Nassar, M. Sbeih, M. Jachiet, Z. Wang, S. Aractingi, Nat. Commun. 2017, 8, 15463.

[51] J. Zhao, Y. C. Liu, Y. H. Shi, Y. Q. Xie, H. P. Cui, Y. Li, X. J. Li, L. Q. Ren, Exp. Ther. Med. 2016, 12, 945.

[52] X. Jiang, L. Huang, D. Xing, Cell. Signalling 2015, 27, 1265. 